MODELING, IDENTIFICATION AND CONTROL, 1996, vOL. 17, NO. 1, 47-61

doi:10.4173/mic.1996.1.5

\title{
Robust adaptive control of underwater vehicles: a comparative study
}

\author{
THOR I. FOSSEN† and OLA-ERIK FJELLSTAD $†$
}

Keywords: $R O V, A U V$, adaptive control, nonlinear velocity observer, marine systems

\begin{abstract}
Robust adaptive control of underwater vehicles in $6 \mathrm{DOF}$ is analysed in the context of measurement noise. The performance of the adaptive control laws of Sadegh and Harowitz (1990) and Slotine and Benedetto (1990) are compared. Both these schemes require that all states are measured, that is the velocities and positions in surge, sway, heave, roll, pitch and yaw. However, for underwater vehicles it is difficult to measure the linear velocities whereas angular velocity measurements can be obtained by using a 3 -axes angular rate sensor. This problem is addressed by designing a nonlinear observer for linear velocity state estimation. The proposed observer requires that the position and the attitude are measured, e.g. by using a hydroacoustic positioning system for linear positions, two gyros for roll and pitch and a compass for yaw. In addition angular rate measurements will be assumed available from a 3-axes rate sensor or a state estimator. It is also assumed that the measurement rate is limited to $2 \mathrm{~Hz}$ for all the sensors. Simulation studies with a 3 DOF AUV model are used to demonstrate the convergence and robustness of the adaptive control laws and the velocity state observer.
\end{abstract}

\section{Introduction}

The implementation of advanced adaptive control laws for underwater vehicles in 6 degrees of freedom (DOF) is mainly limited by the performance of the underwater navigation and sensor systems. The surge, sway and heave position can be measured with fair accuracy by applying a hydroacoustic short or long base-line system whereas attitude usually is measured by 2 gyros (or inclinometers) and a compass. The bandwidth limitation of the control system is mainly due to the hydroacoustic positioning system which usually operates in the interval of $0 \cdot 1-1 \cdot 0 \mathrm{~Hz}$.

This paper discusses the performance of two state-of-the-art adaptive control laws for robot manipulators which are modified to control an underwater vehicle in $6 \mathrm{DOF}$. In addition, a globally asymptotically stable velocity observer using linear acceleration and position measurements is suggested for estimation of linear velocity.

\section{Underwater vehicle model}

The following 6 DOF autonomous underwater vehicle (AUV) equations of motion are considered, Fossen (1994)

$$
M \dot{v}+C(v) v+D(v) v+g(\eta)=\tau
$$

Received 20 August 1995.

$\dagger$ University of Trondheim, The Norwegian Institute of Technology, Department of Engineering Cybernetics, N-7034 Trondheim, Norway (E-mail:tif@itk.unit.no).

Presented at the 3rd IFAC Workshop on Control Applications in Marine Systems, May 1995, Trondheim. Norway (CAMS '95). 


$$
\dot{\boldsymbol{\eta}}=\boldsymbol{J}(\boldsymbol{\eta}) \boldsymbol{v}
$$

where $\boldsymbol{\eta}=[x, y, z, \phi, \theta, \psi]^{\mathrm{T}}$ is the earth-fixed position and attitude vector, $\boldsymbol{v}=[u, v, w, p, q, r]^{\mathrm{T}}$ is a vector of body-fixed linear and angular velocities and $\tau=\left[\tau_{1}, \ldots, \tau_{6}\right]^{\mathrm{T}}$ is a vector of control inputs (force and moment). The matrices and vectors are given in Appendix A.

\section{A globally asymptotically stable velocity observer}

For underwater vehicles it is difficult to measure the linear velocity with good accuracy. A globally asymptotically stable observer can be designed for this purpose. Let

$$
\begin{aligned}
& \boldsymbol{v}=[u, v, w]^{\mathrm{T}} \quad \boldsymbol{x}=[x, y, z]^{\mathrm{T}} \\
& \omega=[p, q, r]^{\mathrm{T}} \quad \boldsymbol{q}=[\phi, \theta, \psi]^{\mathrm{T}}
\end{aligned}
$$

In some cases it is advantageous to use 3 linear accelerometers to improve the convergence of the observer. The location of the linear accelerometer is given by the coordinates

$$
\boldsymbol{r}_{a}=\left[x_{a}, y_{a}, z_{a}\right]^{\mathrm{T}}
$$

\subsection{Estimation of linear velocity}

The output from the linear accelerometers are

$$
a=\dot{v}+\omega \times v+\dot{\omega} \times r_{a}+\omega \times\left(\omega \times r_{a}\right)+g_{1}(q)
$$

where $g_{1}(q)$ is the acceleration of gravity defined as; see (58) in Appendix A

$$
\boldsymbol{g}_{1}(\boldsymbol{q})=[\mathrm{gs} \theta,-g \mathrm{c} \theta \mathrm{s} \phi,-g \mathrm{c} \theta \mathrm{c} \phi]^{\mathrm{T}}
$$

The body-fixed acceleration $\dot{v}$ in (5) can be written in terms of the acceleration measurement $\boldsymbol{a}$ according to

$$
\begin{aligned}
\dot{\boldsymbol{v}} & =-\boldsymbol{S}(\boldsymbol{\omega}) \boldsymbol{v}+\boldsymbol{a}-\boldsymbol{a}_{c} \\
\dot{\boldsymbol{x}} & =J_{1}(\boldsymbol{q}) \boldsymbol{v}
\end{aligned}
$$

where $\boldsymbol{a}_{c}$ is an acceleration correction term defined as

$$
\boldsymbol{a}_{c}=\dot{\boldsymbol{\omega}} \times \boldsymbol{r}_{a}+\omega \times\left(\omega \times \boldsymbol{r}_{a}\right)+\boldsymbol{g}_{1}(\boldsymbol{q})
$$

Eqns. (7) and (8) suggest the observer structure:

$$
\begin{gathered}
\hat{\hat{v}}=-S(\omega) \hat{v}+a-a_{c}+K_{1} \tilde{x} \\
\hat{x}=J_{1}(q) \hat{v}+K_{2} \tilde{x}
\end{gathered}
$$

where $\tilde{\boldsymbol{x}}=\boldsymbol{x}-\hat{\boldsymbol{x}}$ is the position estimation error, and $\boldsymbol{K}_{1}$ and $\boldsymbol{K}_{2}$ are the estimator gain matrices.

\subsection{Computation of $\boldsymbol{K}_{1}$ and $\boldsymbol{K}_{2}$}

Let $\tilde{\boldsymbol{v}}=\boldsymbol{v}-\hat{\boldsymbol{v}}$ and $\tilde{\boldsymbol{x}}=\boldsymbol{x}-\hat{\boldsymbol{x}}$. 


\subsubsection{Kalman filter approach}

Equations (10) and (11) can be written in state-space form

$$
\begin{aligned}
& {\left[\begin{array}{c}
\hat{v} \\
\hat{x}
\end{array}\right]=\left[\begin{array}{cc}
-S(\omega) & 0 \\
J_{1}(q) & 0
\end{array}\right]\left[\begin{array}{l}
\hat{v} \\
\hat{x}
\end{array}\right]} \\
& +\left[\begin{array}{l}
\boldsymbol{I} \\
\mathbf{0}
\end{array}\right] \boldsymbol{u}+\left[\begin{array}{l}
\boldsymbol{K}_{1} \\
\boldsymbol{K}_{2}
\end{array}\right] \tilde{\boldsymbol{y}} \\
& \Uparrow \\
& \dot{\hat{\xi}}(t)=\boldsymbol{A}(t) \hat{\xi}+\boldsymbol{B} u(t)+\boldsymbol{K}(t) \tilde{y}(t)
\end{aligned}
$$

with obvious definitions of $\boldsymbol{A}(t), \boldsymbol{B}$ and $\boldsymbol{K}$, and $\boldsymbol{u}=\boldsymbol{a}-\boldsymbol{a}_{c}, \hat{\boldsymbol{\xi}}=\left[\hat{\boldsymbol{v}}^{\mathrm{T}}, \hat{\boldsymbol{x}}^{\mathrm{T}}\right]^{\mathrm{T}}$ and $\tilde{\boldsymbol{y}}=\boldsymbol{H}(\boldsymbol{\xi}-\hat{\boldsymbol{\xi}})$ with $\boldsymbol{H}=[\mathbf{0}, \boldsymbol{I}]$. Since (14) is a linear time-varying system, $\boldsymbol{K}_{1}$ and $\boldsymbol{K}_{2}$ can be computed by applying the Kalman filter algorithm, Gelb et al. (1988)

$$
\boldsymbol{K}(t)=\boldsymbol{P}(t) \boldsymbol{H}^{\mathrm{T}} \boldsymbol{R}^{-1}
$$

with

$$
\begin{aligned}
\dot{\hat{\xi}}(t) & =A(t) \hat{\xi}(t)+\boldsymbol{B} u(t)+\boldsymbol{K}(t) \tilde{x}(t) \\
\dot{\boldsymbol{P}}(t) & =\boldsymbol{A}(t) \boldsymbol{P}(t)+\boldsymbol{P}(t) \boldsymbol{A}^{\mathrm{T}}(t)+\boldsymbol{Q}-\boldsymbol{K}(t) \boldsymbol{R} \boldsymbol{K}^{\mathrm{T}}(t)
\end{aligned}
$$

where $\boldsymbol{Q}$ is the process and $\boldsymbol{R}$ is the measurement covariance matrices. This requires that the time-varying error covariance matrix propagation given by (17) must be computed on-line.

\subsubsection{Nonlinear observer design}

An alternative approach to the Kalman filter algorithm is a nonlinear pole-placement algorithm utilizing the structure of the observer dynamics. Notice that

$$
\dot{J}_{1}(q)=J_{1}(q) S(\omega)
$$

Hence (10) and (11) can be written as

$$
\ddot{\tilde{x}}(t)+K_{2} \dot{\tilde{x}}(t)+J_{1}(q) K_{1} \tilde{x}(t)=\mathbf{0}
$$

An intuitive solution is to choose $\boldsymbol{K}_{1}$ and $\boldsymbol{K}_{2}$ as

$$
\boldsymbol{K}_{1}=\boldsymbol{J}_{1}^{\mathrm{T}}(\boldsymbol{q}) \boldsymbol{K}_{10} ; \quad \boldsymbol{K}_{2}=\boldsymbol{K}_{20}
$$

which reduces (19) to

$$
\ddot{\tilde{\boldsymbol{x}}}(t)+\boldsymbol{K}_{20} \dot{\tilde{\boldsymbol{x}}}(t)+\boldsymbol{K}_{10} \tilde{\boldsymbol{x}}(t)=\mathbf{0}
$$

Eqn. (21) satisfies the Lyapunov function candidate

$$
\begin{aligned}
& V=\frac{1}{2}\left(\dot{\tilde{\boldsymbol{x}}}^{\mathrm{T}} \dot{\tilde{\boldsymbol{x}}}+\tilde{\boldsymbol{x}}^{\mathrm{T}} \boldsymbol{K}_{10 \tilde{\boldsymbol{x}}} \tilde{)}\right. \\
& \Downarrow \\
& \dot{\boldsymbol{V}}=-\dot{\tilde{\boldsymbol{x}}}^{\mathrm{T}} \boldsymbol{K}_{10} \dot{\tilde{\boldsymbol{x}}} \leqslant 0
\end{aligned}
$$

Application of LaSalle's invariant set theorem then shows that $\dot{\tilde{\boldsymbol{x}}} \rightarrow \mathbf{0}$ and $\tilde{\boldsymbol{x}} \rightarrow \mathbf{0}$ in finite time. 


\subsection{Implementation issues}

Implementation of (10) and (11) requires that $x, q, \omega$ and $a$ are measured. If acceleration $\boldsymbol{a}$ is inconvenient to measure the observer structure in Fig. 1 can be modified to use an estimate of $a$ generated by a filter, e.g. (see Fig..3)

$$
\dot{a}=-\frac{1}{T} a+K_{0} \tilde{x} ; \quad K_{0}=\frac{1}{T} K_{00}>0
$$

where $T>0$ is the low-pass filter time constant reflecting the vehicle dynamics.

If $\omega$ is not measured, $\omega$ should be approximated by $\tilde{\omega}$ which can be obtained from an angular velocity observer. Assuming that $\dot{\omega} \approx 0$ (slowly-varying attitude) yield

$$
\boldsymbol{a}_{c} \approx \hat{\boldsymbol{\omega}} \times\left(\hat{\boldsymbol{\omega}} \times \boldsymbol{r}_{a}\right)+\boldsymbol{g}_{1}(\boldsymbol{q})
$$

\subsection{Simulation study of observer}

The nonlinear observer (10) and (11) was simulated with $\boldsymbol{a}_{c}=\mathbf{0}$. The AUV model in Appendix B was used to generate the acceleration measurements in surge and sway while the heave acceleration was set to zero. The heading of the vehicle was chosen as

$$
\psi(t)=0.5 \sin (0.03 t) ; \quad r(t)=0.015 \cos (0.03 t)
$$

The estimator gain matrices were chosen as $\boldsymbol{K}_{10}=0.001 \boldsymbol{I}$ and $\boldsymbol{K}_{20}=1.0 I$. The sampling frequency was $2 \mathrm{~Hz}$. It is seen from Fig. 2 that accurate estimates of $u$ and $v$ can be generated from noisy position measurements (approx. amplitude of the white noise is $\pm 1.0 \mathrm{~m})$.

\section{Adaptive control}

The stability proof and representation of the two control laws to be presented are based on the parametrization

$$
M \dot{r}+C(v) r+D(v) r \triangleq Y(\dot{r}, r, v) \theta
$$

The gravity and buoyancy term $\boldsymbol{g}(\boldsymbol{\eta})$ (without loss of generality) is assumed to be perfectly known. In this representation $\boldsymbol{Y}(\dot{\boldsymbol{r}}, \boldsymbol{r}, \boldsymbol{v})$ is the regressor matrix (known) and $\boldsymbol{\theta}$ is a constant parameter vector (unknown). Let the tracking error be denoted by

$$
\tilde{\boldsymbol{\eta}}=\boldsymbol{\eta}-\boldsymbol{\eta}_{d}
$$

where $\boldsymbol{\eta}_{d} \in \mathbb{R}^{6}$ is assumed to be a smooth reference trajectory.

\subsection{The adaptive control law of Slotine and Benedetto (ASB)}

The adaptive control law of Slotine and Benedetto (1990) with the parametrization of Fossen (1993) is actually intended for 3 DOF spacecraft attitude control. An extension of this work to 6 DOF underwater vehicle control is discussed by Fossen and Sagatun (1991). The ASB adaptive control scheme can be represented in terms of the model parametrization (P), control law (C) and parameter adaptation law (A) according to

$$
\begin{array}{ll}
\text { P: } & \boldsymbol{M} \dot{v}_{r}+C(v) v_{r}+D(v) v_{r} \triangleq Y\left(\dot{v}_{r}, v_{r}, v\right) \theta \\
\text { C: } \quad \tau=\boldsymbol{Y}\left(\dot{v}_{r}, v_{r}, v\right) \hat{\theta}+g(v)-J^{\mathrm{T}}(\eta) K_{d} s \\
\text { A: } \quad \dot{\hat{\theta}}=-\Gamma \boldsymbol{Y}^{\mathrm{T}}\left(\dot{v}_{r}, v_{r}, v\right) J^{\mathrm{j}-1}(\eta) s
\end{array}
$$




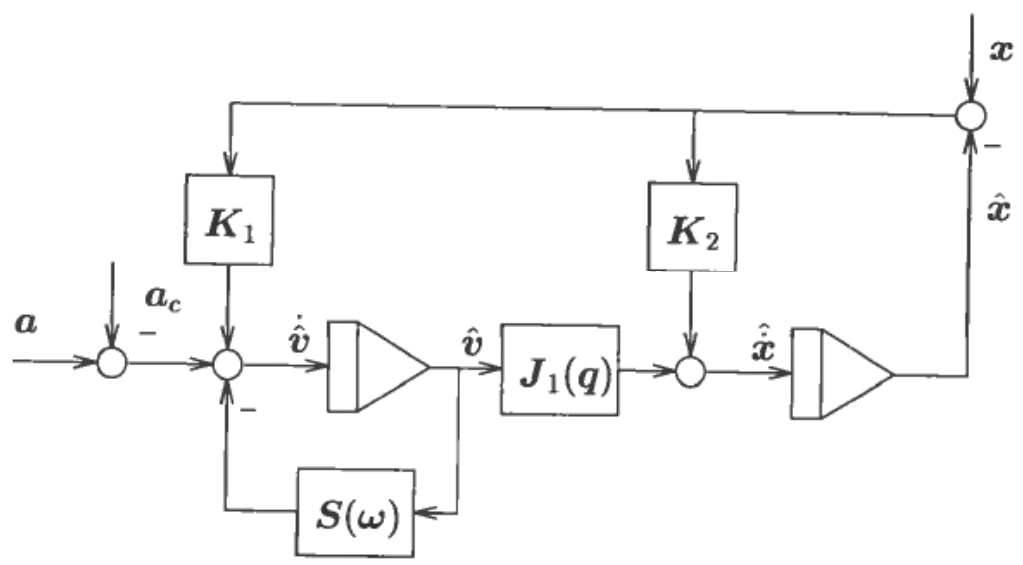

Figure 1. Nonlinear velocity observer using position and linear acceleration measurements.
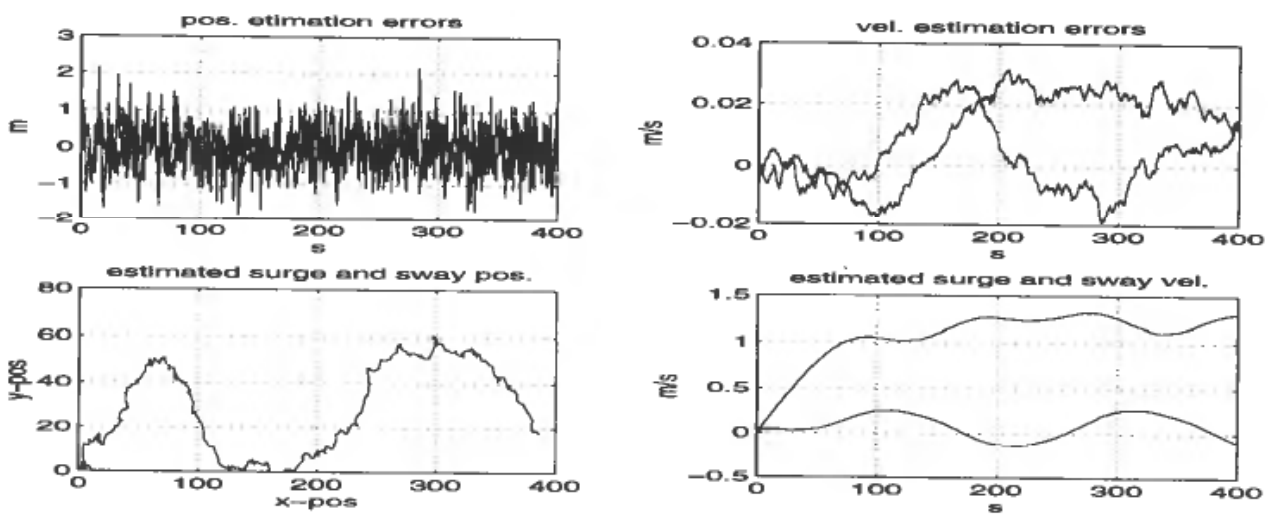

Figure 2. Position and velocity estimation errors (upper plots) and position and velocity state estimates (lower plots).

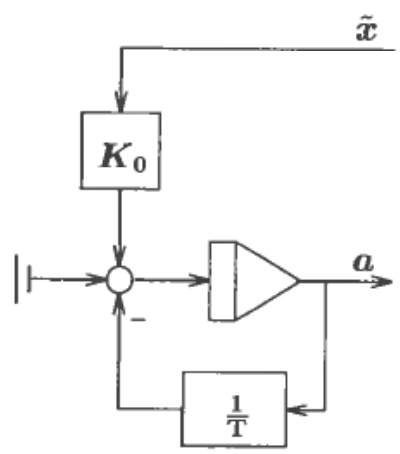

Figure 3. Estimation of acceleration $\boldsymbol{a}$ in Fig. 1. $\boldsymbol{K}_{0}$ is a new filter gain matrix to be computed together with $K_{1}$ and $K_{2}$ in Fig. 1. 
where $\boldsymbol{K}_{d}>\mathbf{0}, \boldsymbol{\Gamma}=\boldsymbol{\Gamma}^{\mathrm{T}}>\mathbf{0}$ and

$$
\boldsymbol{s}=\dot{\tilde{\boldsymbol{\eta}}}+\boldsymbol{\Lambda} \tilde{\boldsymbol{\eta}} ; \quad \boldsymbol{\Lambda}=\boldsymbol{\Lambda}^{\mathrm{T}}>\mathbf{0}
$$

The virtual reference trajectories are computed according to

$$
\begin{aligned}
& \boldsymbol{v}_{r}=J^{-1}(\boldsymbol{\eta}) \dot{\boldsymbol{\eta}}_{r} \\
& \dot{\boldsymbol{v}}_{r}=\boldsymbol{J}^{-1}(\boldsymbol{\eta})\left[\ddot{\boldsymbol{\eta}}_{r}-\dot{\boldsymbol{J}}(\boldsymbol{\eta}) \boldsymbol{J}^{-1}(\boldsymbol{\eta}) \dot{\boldsymbol{\eta}}_{r}\right]
\end{aligned}
$$

where $\dot{\boldsymbol{\eta}}_{r}=\dot{\boldsymbol{\eta}}_{d}-\Lambda \tilde{\boldsymbol{\eta}}$.

\subsection{The adaptive control law of Sadegh and Horowitz (DCAL)}

Straightforward extensions of the robot control scheme of Sadegh and Horowitz (1990) to underwater vehicles yields

$$
\begin{array}{ll}
\text { P: } & \boldsymbol{M} \dot{\boldsymbol{v}}_{d}+\boldsymbol{C}\left(\boldsymbol{v}_{d}\right) \boldsymbol{v}_{d}+\boldsymbol{D}\left(\boldsymbol{v}_{d}\right) \boldsymbol{v}_{d} \triangleq \boldsymbol{Y}\left(\dot{\boldsymbol{v}}_{d}, \boldsymbol{v}_{d}\right) \boldsymbol{\theta} \\
\mathrm{C}: & \tau=\boldsymbol{Y}\left(\dot{\boldsymbol{v}}_{d}, \boldsymbol{v}_{d}\right) \hat{\boldsymbol{\theta}}+\boldsymbol{g}\left(\boldsymbol{\eta}_{d}\right)-\boldsymbol{J}^{\mathrm{T}}(\boldsymbol{\eta})\left[K_{p} \tilde{\boldsymbol{\eta}}+\boldsymbol{K}_{d} \dot{\tilde{\eta}}+K_{f}\|\tilde{\boldsymbol{\eta}}\|^{2} \boldsymbol{s}\right] \\
\text { A: } & \dot{\hat{\boldsymbol{\theta}}}=-\boldsymbol{\Gamma} \boldsymbol{Y}^{\mathrm{T}}\left(\dot{\boldsymbol{v}}_{d}, \boldsymbol{v}_{d}\right) \boldsymbol{J}^{-1}(\boldsymbol{\eta}) \boldsymbol{s}
\end{array}
$$

where $\boldsymbol{K}_{p}=\boldsymbol{K}_{p}^{\mathrm{T}}>\mathbf{0}, \boldsymbol{K}_{d}>\mathbf{0}, \boldsymbol{K}_{f}>\mathbf{0}, \boldsymbol{\Gamma}=\boldsymbol{\Gamma}^{\mathrm{T}}>\mathbf{0}$. The above control law is known as the desired compensation adaptive law (DCAL) since the desired states $v_{d}$ and $\eta_{d}$ are used instead of the actual states $\boldsymbol{v}$ and $\boldsymbol{\eta}$ in $\boldsymbol{C}\left(\boldsymbol{v}_{d}\right), \boldsymbol{D}\left(\boldsymbol{v}_{d}\right)$ and $\boldsymbol{g}\left(\boldsymbol{\eta}_{d}\right)$.

\subsection{AUV case study}

The performance of the ASB and DCAL control laws are studied for the NPS AUV II given in Appendix B. A combined velocity and position control scheme is studied for the following cases:

1. Parameter adaptation, ASB

2. All parameters known, ASB

3. Parameter adaptation, DCAL

4. All parameters known, DCAL

The results are presented according to:

- Section 4.3.1: Perfect measurements

- Section 4.3.2: Noisy measurements

The performance indices are chosen as

$$
\begin{aligned}
J_{u v} & =\frac{1}{N} \sum_{i=1}^{N}\left[u\left(t_{i}\right)-u_{d}\left(t_{i}\right)\right]^{2}+\left[v\left(t_{i}\right)-v_{d}\left(t_{i}\right)\right]^{2} \\
J_{r} & =\frac{1}{N} \sum_{i=1}^{N}\left[r\left(t_{i}\right)-r_{d}\left(t_{i}\right)\right]^{2} \\
J_{\psi} & =\frac{1}{N} \sum_{i=1}^{N}\left[\phi\left(t_{i}\right)-\phi_{d}\left(t_{i}\right)\right]^{2} \\
J_{\tau} & =\frac{1}{N} \sum_{i=1}^{N} \tau^{\mathrm{T}}\left(t_{i}\right) \tau\left(t_{i}\right)
\end{aligned}
$$




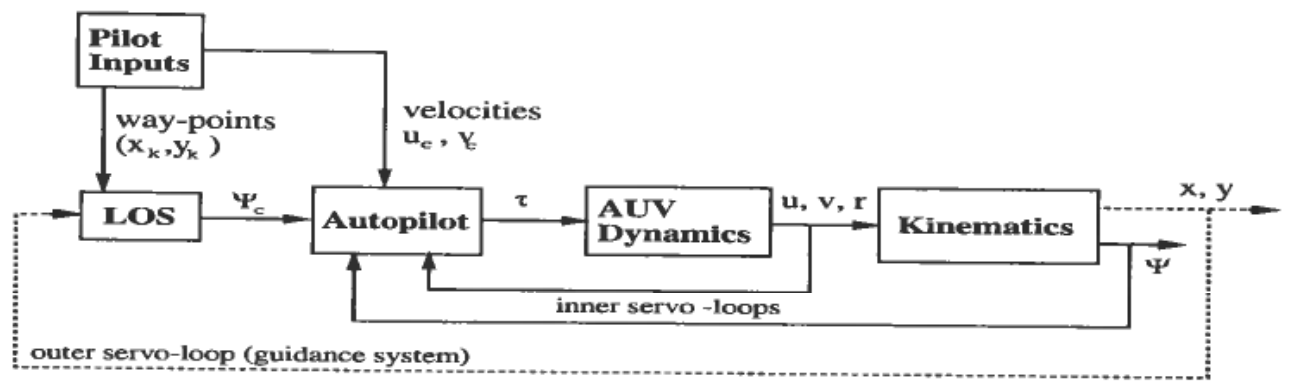

Figure 4. Way-point guidance by means of a yaw angle autopilot and a surge-sway velocity control system. The line-of-sight (LOS) algorithm is used to generate the desired yaw angle.

where $N$ is the number of samples used in the simulation study. The sampling frequency is chosen as $2 \mathrm{~Hz}$. The DCAL and ASB control laws were tuned according to

$$
\begin{aligned}
\boldsymbol{K}_{d}^{A S B} & =\boldsymbol{K}_{D} \\
\boldsymbol{K}_{p}^{D C A L} & =\boldsymbol{\Lambda} \boldsymbol{K}_{D} \\
\boldsymbol{K}_{d}^{D C A L} & =\boldsymbol{K}_{D} \\
\boldsymbol{K}_{f}^{D C A L} & =\mathbf{0}
\end{aligned}
$$

with $\Lambda=0 \cdot 1 I$ and $K_{D}=5000 I$. An autopilot for simultaneously heading and speed control (surge and sway) was considered, see Fig. 4. The desired heading states $\left(\ddot{\psi}_{d,}, \dot{\psi}_{d}, \psi_{d}\right)$, surge states $\left(\dot{u}_{d}, u_{d}\right)$ and sway states $\left(\dot{v}_{d}, v_{d}\right)$ are computed by using the filters

$$
\begin{aligned}
\ddot{\psi}_{d}+2 \zeta \omega_{n} \dot{\psi}_{d}+\omega_{n}^{2} \psi_{d} & =\omega_{n}^{2} \psi_{f} \\
T \dot{u}_{d}+u_{d} & =u_{f} \\
T \dot{v}_{d}+v_{d} & =v_{f}
\end{aligned}
$$

where the reference inputs $\psi_{f}, u_{f}$ and $v_{f}$ are pre-filtered by a 1st-order filter to avoid acceleration step inputs. The parameters were chosen as $\zeta=1 \cdot 0, \omega_{n}=0 \cdot 1 \mathrm{rad} / \mathrm{s}$ and $T=1 / \omega_{n}=10 \cdot 0 \mathrm{~s}$. The pre-filters are designed according to

$$
\begin{aligned}
T_{f} \dot{u}_{f}+u_{f} & =u_{c} \\
T_{f} \dot{v}_{f}+v_{f} & =v_{c} \\
T_{f} \dot{\psi}_{f}+\psi_{f} & =\psi_{c}
\end{aligned}
$$

where $T_{f}=T$, and $u_{c}, v_{c}$ and $\psi_{c}$ are the commanded surge velocity, sway velocity and yaw angle, respectively. The commanded input $\psi_{c}$ can be computed by applying a line-of-sight (LOS) algorithm, e.g. Healey and Lienard (1993)

$$
\psi_{c}(t)=\tan ^{-1}\left(\frac{y_{d}(k)-y(t)}{x_{d}(k)-x(t)}\right)
$$

where $x_{k}$ and $y_{k}(k=1 \ldots N)$ represents the way-point coordinates. Care must be taken to select the proper quadrant for $\psi_{c}(t)$. After the quadrant check is performed, the next way point can be selected on a basis of whether the vessel lies within a circle of acceptance

$$
\left[x_{d}(k)-x(t)\right]^{2}+\left[y_{d}(k)-y(t)\right]^{2} \leqslant \rho_{0}^{2}
$$


where $\rho_{0}$ is the radius. If this constraint is satisfied the next way point $\left[x_{d}(k+1)\right.$, $\left.y_{d}(k+1)\right]$ should be selected. The measure of tracking is

$$
\boldsymbol{s}=[\tilde{u}, \tilde{v}, \dot{\bar{\psi}}+\lambda \tilde{\psi}]^{\mathrm{T}}
$$

Hence the ASB virtual reference trajectories can be computed as

$$
\dot{v}_{r}=\left[\begin{array}{c}
\dot{u}_{r} \\
\dot{v}_{r} \\
\ddot{\psi}_{r}
\end{array}\right]=\left[\begin{array}{c}
u_{d} \\
v_{d} \\
\ddot{\psi}_{d}-\lambda\left(\dot{\psi}-\dot{\psi}_{d}\right)
\end{array}\right]
$$

The resulting control laws are

$$
\begin{aligned}
\tau^{A S B} & =\boldsymbol{Y}\left(\dot{\boldsymbol{v}}_{r}, \boldsymbol{v}_{r}, \boldsymbol{v}\right) \hat{\boldsymbol{\theta}}^{A S B}-\boldsymbol{K}_{D} \boldsymbol{S} \\
\tau^{D C A L} & =\boldsymbol{Y}\left(\dot{\boldsymbol{v}}_{d}, \boldsymbol{v}_{d}, \boldsymbol{v}\right) \hat{\boldsymbol{\theta}}^{D C A L}-\boldsymbol{K}_{D} \boldsymbol{S}
\end{aligned}
$$

where $\boldsymbol{Y}(\cdot)$ is given in Appendix B and

$$
\begin{aligned}
\hat{\boldsymbol{\theta}}^{A S B} & =-\boldsymbol{\Gamma} \boldsymbol{Y}^{\mathrm{T}}\left(\boldsymbol{v}_{r}, \boldsymbol{v}_{r}, \boldsymbol{v}\right) \boldsymbol{s} \\
\dot{\boldsymbol{\theta}}^{D C A L} & =-\boldsymbol{\Gamma} \boldsymbol{Y}^{\mathrm{T}}\left(\dot{\boldsymbol{v}}_{d}, \boldsymbol{v}_{d}, \boldsymbol{v}_{d}\right) \boldsymbol{S}
\end{aligned}
$$

where $\boldsymbol{\Gamma}=100 \boldsymbol{I}$. Notice that $\boldsymbol{J}(\boldsymbol{\eta})=\boldsymbol{I}$ for this particular case since $\dot{\psi}=r$ whereas the other two states are velocity controlled.

\subsubsection{Perfect measurements}

The numerical results are shown in Table 1. Figure 5 shows the parameter estimates and Fig. 6 shows the tracking performance of the two algorithms. Notice that the performance of the two adaptive controllers is almost equal. Also notice that the perfect model control laws $\left(\boldsymbol{\theta}=\boldsymbol{\theta}_{\text {true }}\right)$ yield better performance than their adaptive counterparts.

\subsubsection{Noisy measurements}

The simulation study in Section 4.3.1 was repeated by adding white noise to the state measurements of $u, v, r$ and $\psi$. It turned out the ASB algorithm was quite sensitive to measurement noise. For instance adding a zero-mean white noise signal $\eta_{\psi}$ to the yaw angle measurement:

$$
\psi_{m}=\psi+\eta_{\psi} ; \quad\left|\eta_{\psi}\right| \leqslant 1.0 \mathrm{deg}
$$

gave the parameter estimates shown in Fig. 7. It is seen that for the ASB algorithm, the parameter estimates of $\theta_{3}=I_{z}-N_{r}$ and $\theta_{6}=-N_{r}$ both corresponding to the yaw mode are drifting. It is also seen that the DCAL parameter estimates are bounded even for the noisy case.

Parameter drift in the ASB algorithm can be explained by considering the update laws for $\theta_{3}$ and $\theta_{6}$ where the square signal $\psi_{m}^{2}$ occurs. This implies that white noise $\eta_{\psi}$ is squared and integrated up to a non-zero term causing parameter drift. For the DCAL algorithm this is avoided since the product between the desired state $\psi_{d}$ and the measurement $\psi_{m}$ is used instead. The performance of the DCAL algorithm with the highly noise contaminated signals:

$$
\begin{array}{ll}
u_{m}=u+\eta_{u} ; & \left|\eta_{u}\right| \leqslant 0 \cdot 1 \mathrm{~m} / \mathrm{s} \\
v_{m}=v+\eta_{v} ; & \left|\eta_{v}\right| \leqslant 0 \cdot 1 \mathrm{~m} / \mathrm{s}
\end{array}
$$




\begin{tabular}{|c|c|c|c|c|c|}
\hline \multirow[b]{2}{*}{ Index } & \multicolumn{2}{|c|}{ ASB } & \multicolumn{2}{|c|}{ DCAL } & \multirow[b]{2}{*}{ Units } \\
\hline & 1. Adaptive & $\begin{array}{l}\text { 2. Perfect } \\
\text { model }\end{array}$ & 3. Adaptive & $\begin{array}{l}\text { 4. Perfect } \\
\text { model }\end{array}$ & \\
\hline$J_{u v}$ & 0.0011 & $3.9892 \cdot 10^{-8}$ & 0.0011 & $6.5414 \cdot 10^{-6}$ & $(\mathrm{~m} / \mathrm{s})^{2}$ \\
\hline$J_{r}$ & 0.0003 & $9 \cdot 5260 \cdot 10^{-6}$ & $0 \cdot 0003$ & $1 \cdot 3727 \cdot 10^{-5}$ & $(\mathrm{deg} / \mathrm{s})^{2}$ \\
\hline$J_{\psi}$ & 0.1249 & 0.0009 & $0 \cdot 1021$ & 0.0015 & $(\mathrm{deg})^{2}$ \\
\hline $\boldsymbol{J}_{r}$ & 3.9795 & 4.5612 & 3.9753 & 4.5633 & $10^{6}$ \\
\hline
\end{tabular}

Table 1. Performance indices for the ASB and DCAL control laws. Full state feedback and no measurement noise (800 samples).

\begin{tabular}{ccccccc}
\hline & \multicolumn{2}{c}{ ASB } & & \multicolumn{3}{c}{ DCAL } \\
\cline { 2 - 5 } \cline { 5 - 7 } Index & 1. Adaptive & $\begin{array}{c}\text { 2. Perfect } \\
\text { model }\end{array}$ & & 3. Adaptive & $\begin{array}{c}\text { 4. Perfect } \\
\text { model }\end{array}$ & Units \\
\hline$J_{u v}$ & par. drift & 0.0070 & & 0.0067 & 0.0048 & $(\mathrm{~m} / \mathrm{s})^{2}$ \\
$J_{r}$ & par. drift & 0.0278 & & 0.0212 & 0.0189 & $(\mathrm{deg} / \mathrm{s})^{2}$ \\
$J_{\psi}$ & par. drift & 2.1421 & & 2.4389 & 2.4539 & $(\mathrm{deg})^{2}$ \\
$J_{r}$ & par. drift & 8.9384 & & 8.9091 & 8.9012 & $10^{6}$ \\
\hline
\end{tabular}

Table 2. Performance indices for the ASB, DCAL and PD control laws. Full state feedback and measurement noise (800 samples).
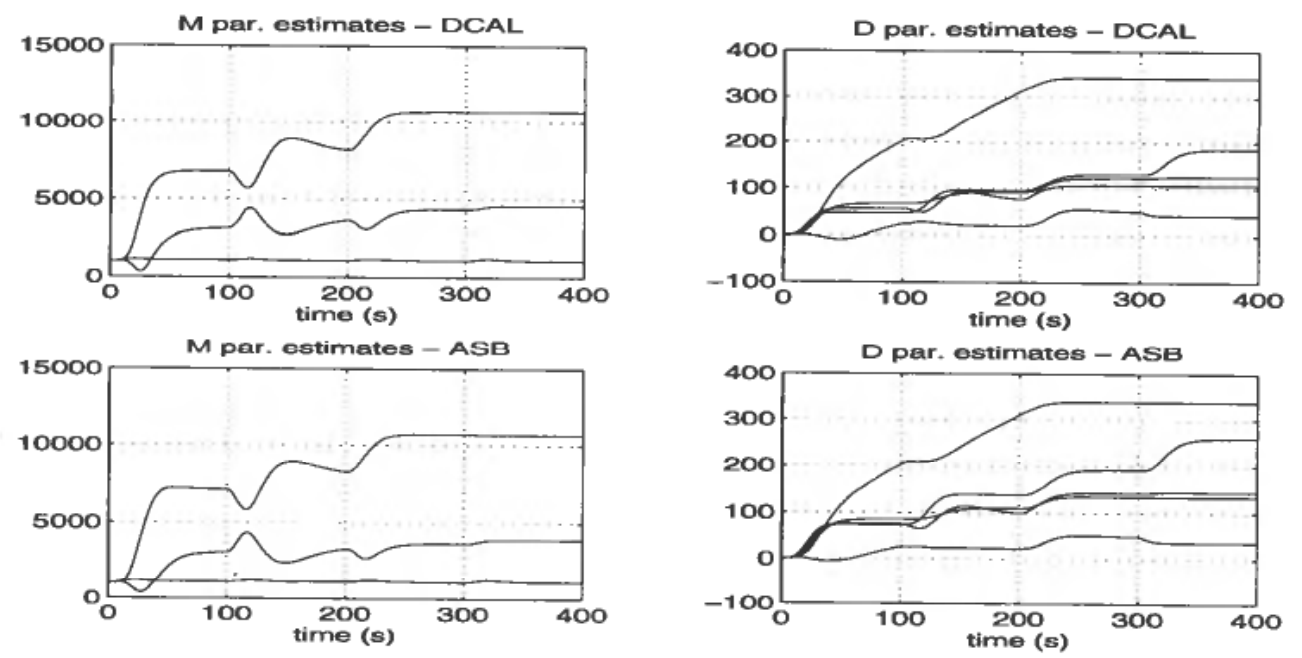

Figure 5. No measurement noise: Parameter estimates for ASB and DCAL.

$$
\begin{array}{ll}
r_{m}=r+\eta_{r} ; & \left|\eta_{r}\right| \leqslant 0 \cdot 1 \mathrm{deg} / \mathrm{s} \\
\psi_{m}=\psi+\eta_{\psi} ; & \left|\eta_{\psi}\right| \leqslant 1.0 \mathrm{deg}
\end{array}
$$

are shown in Fig. 8. It is shown that the performance is satisfactory also for this case. 

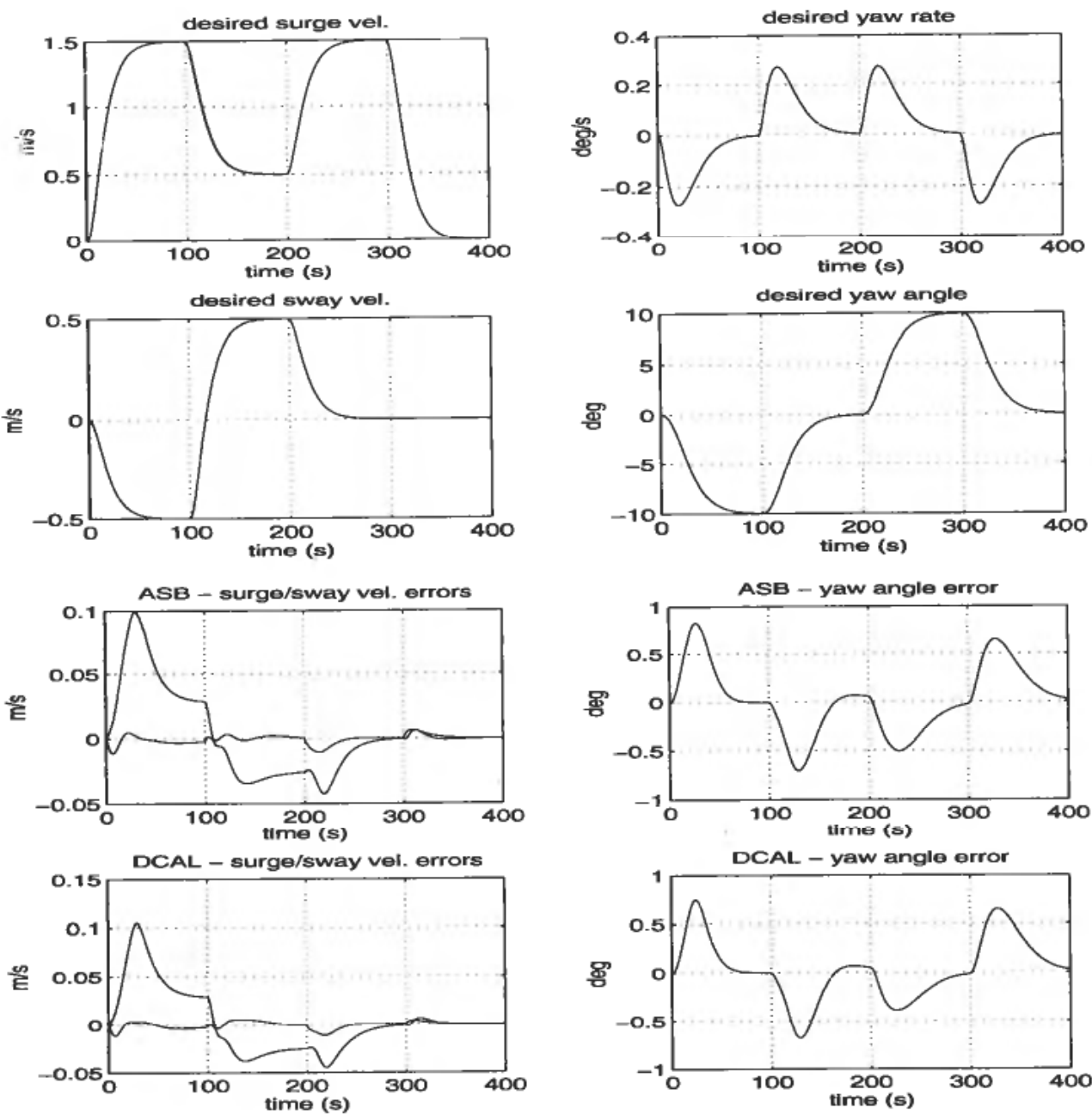

Figure 6. Upper plots show the desired states whereas the lower plots show the tracking errors for the ASB and DCAL (no measurement noise).

\subsection{Comments on the ASB and DCAL algorithms}

It is well known that the ASB control scheme is sensitive to velocity measurement noise, see Berghuis (1993) for instance. For an underwater vehicle body-fixed velocities are usually obtained by model-based state estimation through noisy position measurements. This implies that the velocity estimates can be contaminated with a significant amount of noise. In such cases the ASB algorithm can go unstable due to drift in the parameter estimates. However, some precautions against parameter drift can be taken by small modifications of the adaptive scheme e.g. by applying the so-called $\sigma$ and $e_{1}$ modification schemes, Narendra and Annaswamy (1987).

The case study showed that the DCAL was less sensitive to velocity measurement noise than the ASB. The main reason for this is that the actual position and velocity in the DCAL regressor is replaced by the desired state trajectories. 

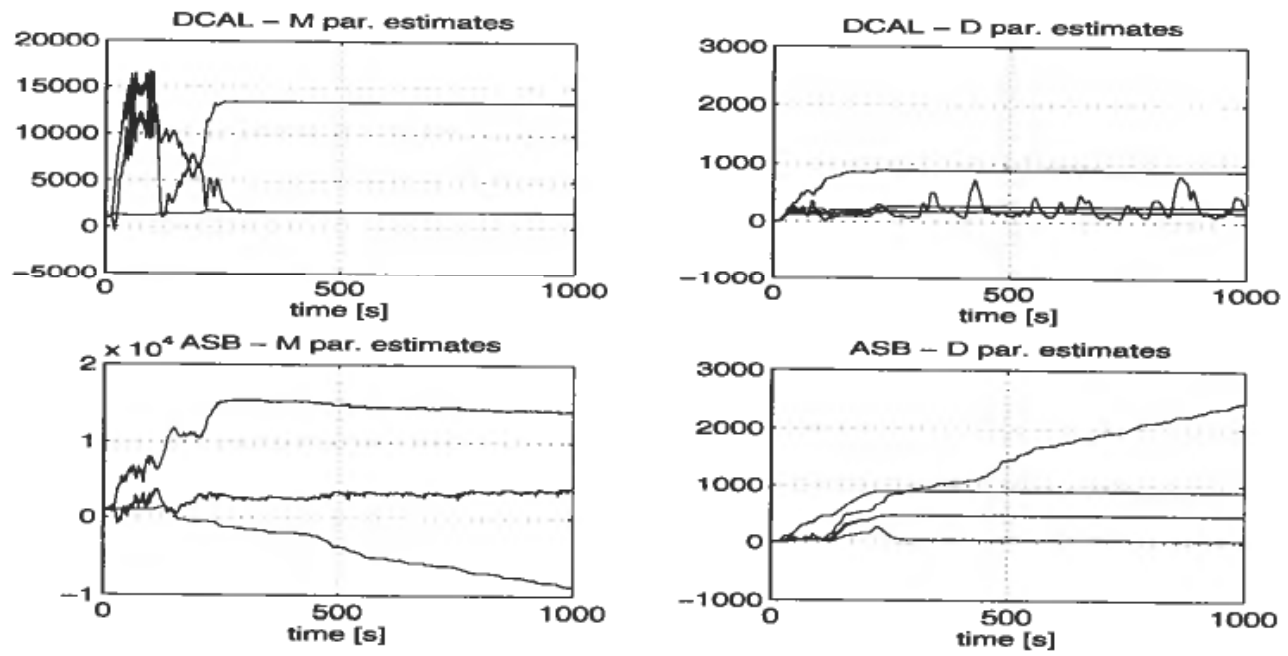

Figure 7. Measurement noise: Mass matrix parameter estimates for ASB and DCAL. Notice that $\theta_{3}$ and $\theta_{6}$ are drifting for the ASB algorithm.
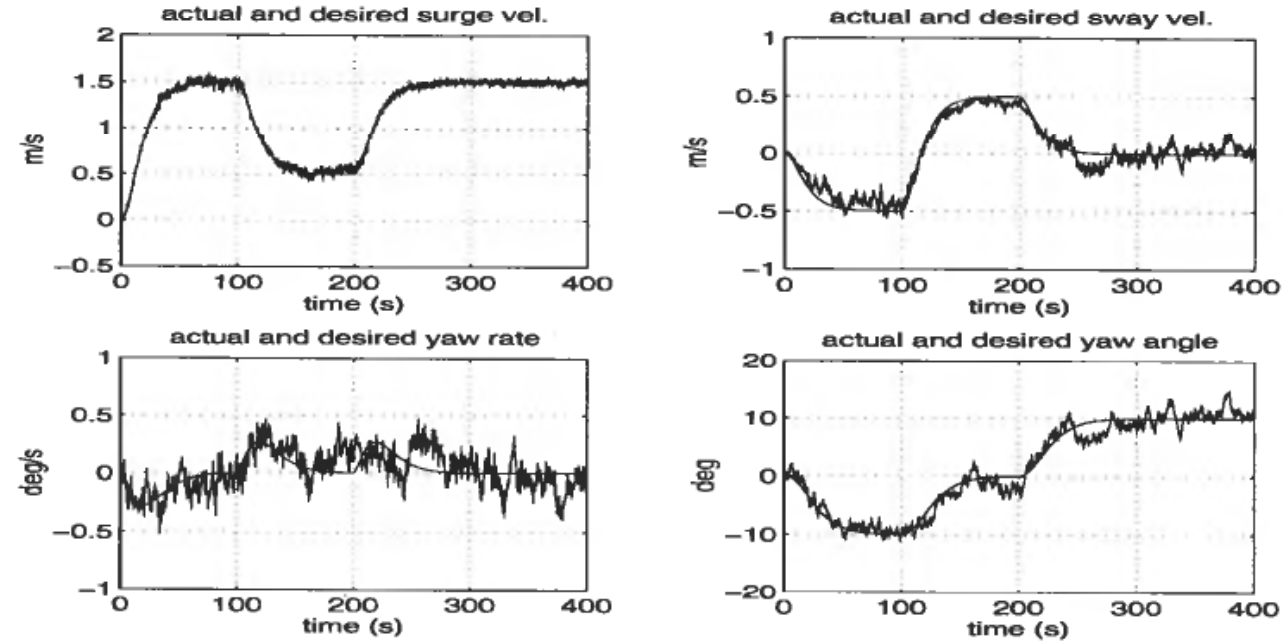

Figure 8. Measurement noise: Actual and desired states for DCAL.

\section{Conclusions}

In this paper two direct adaptive control laws, referred to as the adaptive control law of Slotine and Benedetto (ASB), and the desired compensation adaptive control law (DCAL) by Sadegh and Horowitz, have been studied in the context of measurement noise. It is concluded that the DCAL algorithm is the only suitable algorithm for real-time AUV applications. A globally asymptotically nonlinear observer for estimation of linear velocity is also presented.

The observer is intended for implementation together with the DCAL algorithm. The case study also shows that the DCAL with fixed parameters is highly robust for measurement noise. It can also be concluded that direct parameter adaptation should only be used if accurate position measurements are available at high sampling frequency $(1-10 \mathrm{~Hz})$. 


\section{Appendix A. General 6 DOF AUV model}

$A U V$ dynamics. The $6 \times 6$ inertia matrix $M$ included hydrodynamic added mass is written

$$
M=\left[\begin{array}{ll}
M_{11} & M_{12} \\
M_{21} & M_{22}
\end{array}\right]
$$

Expanding this expression in terms of the SNAME (1950) notation yields

$$
\begin{aligned}
& \boldsymbol{M}=\left[\begin{array}{ccc}
m-X_{\dot{u}} & -X_{\dot{v}} & -X_{\dot{w}} \\
-X_{\dot{v}} & m-Y_{\dot{v}} & -Y_{\dot{w}} \\
-X_{\dot{w}} & -Y_{\dot{w}} & m-Z_{\dot{w}} \\
-X_{\dot{p}} & -m z_{G}-Y_{\dot{p}} & m y_{G}-Z_{\dot{p}} \\
z_{G}-X_{\dot{q}} & -Y_{\dot{q}} & -m x_{G}-Z_{\dot{q}} \\
-m y_{G}-X_{\dot{r}} & m x_{G}-Z_{\dot{r}} & -Z_{\dot{r}}
\end{array}\right. \\
& \left.\begin{array}{ccc}
-X_{\dot{p}} & m z_{G}-X_{\dot{q}} & -m y_{G}-X_{\dot{r}} \\
-m z_{G}-Y_{\dot{p}} & -Y_{\dot{q}} & m x_{G}-Y_{\dot{r}} \\
m y_{G}-Z_{\dot{p}} & -m x_{G}-Z_{\dot{q}} & -Z_{\dot{r}} \\
I_{x}-K_{\dot{p}} & -I_{x y}-K_{\dot{q}} & -I_{z x}-K_{\dot{r}} \\
-I_{x y}-K_{\dot{q}} & I_{y}-M_{\dot{q}} & -I_{y z}-M_{\dot{r}} \\
-I_{z x}-K_{\dot{r}} & -I_{y z}-M_{\dot{r}} & I_{z}-N_{\dot{r}}
\end{array}\right]
\end{aligned}
$$

Sagatun and Fossen (1991) have shown that the Coriolis and centripetal matrix can be defined in terms of the inertia matrix elements according to

$$
C(v)=\left[\begin{array}{cc}
0_{3 \times 3} & -S\left(M_{11} v_{1}+M_{12} v_{2}\right) \\
-S\left(M_{11} v_{1}+M_{12} v_{2}\right. & -S\left(M_{21} v_{1}+M_{22} v_{2}\right)
\end{array}\right]
$$

where $S(\cdot)$ is a $3 \times 3$ skcw-symmetrical matrix operator defined such that $\boldsymbol{S}(\boldsymbol{a}) \boldsymbol{b}=\boldsymbol{a} \times \boldsymbol{b}$ for $\boldsymbol{a} \in \mathbb{R}^{3}$ and $\boldsymbol{b} \in \mathbb{R}^{3}$; see Fossen and Fjellstad (1995) for details. The vector term $D(v) v \in \mathbb{R}^{6}$ is simply a vector containing dissipative forces and moments due to hydrodynamic damping and lift whereas $\boldsymbol{g}(\boldsymbol{\eta}) \in \mathbb{R}^{6}$ is the gravitational and buoyant forces and moments defined by

$$
\begin{aligned}
\boldsymbol{g}(\boldsymbol{\eta}) & =\left[g_{1}^{\mathrm{T}}(\boldsymbol{\eta}), \boldsymbol{g}_{2}^{\mathrm{T}}(\boldsymbol{\eta})\right]^{\mathrm{T}} \\
= & {\left[\begin{array}{l}
(W-B) \mathrm{s} \theta \\
-(W-B) \mathrm{c} \theta \mathrm{s} \phi \\
-(W-B) \mathrm{c} \theta \mathrm{c} \phi \\
-\left(y_{G} W-y_{B} B\right) \mathrm{c} \theta \mathrm{c} \phi+\left(z_{G} W-z_{B} B\right) \mathrm{c} \theta \mathrm{s} \phi \\
\left(z_{G} W-z_{B} B\right) \mathrm{s} \theta+\left(x_{G} W-x_{B} B\right) \mathrm{c} \theta \mathrm{c} \phi \\
-\left(x_{G} W-x_{B} B\right) \mathrm{c} \theta \mathrm{s} \phi-\left(y_{G} W-y_{B} B\right) \mathrm{s} \theta
\end{array}\right] }
\end{aligned}
$$


Kinematics. Equation (2) describes the kinematic equations of motion where $\boldsymbol{J}(\boldsymbol{\eta})$ is a $6 \times 6$ block diagonal transformation matrix usually defined in terms of the Euler angles $\phi, \theta$ and $\psi$ according to

$$
J(\eta)=\left[\begin{array}{ll}
J_{1}(\eta) & \mathbf{0}_{3 \times 3} \\
\mathbf{0}_{3 \times 3} & J_{2}(\eta)
\end{array}\right]
$$

Here $\boldsymbol{J}_{1}(\boldsymbol{\eta})$ is the Euler angle rotation matrix defined as

$$
\boldsymbol{J}_{1}(\boldsymbol{\eta})=\left[\begin{array}{ccc}
\mathrm{c} \psi \mathrm{c} \theta & -\mathrm{s} \psi \mathrm{c} \phi+\mathrm{c} \psi \mathrm{s} \theta \mathrm{s} \phi & \mathrm{s} \psi \mathrm{s} \phi+\mathrm{c} \psi \mathrm{c} \phi \mathrm{s} \theta \\
\mathrm{s} \psi \mathrm{c} \theta & \mathrm{c} \psi \mathrm{c} \phi+\mathrm{s} \phi \mathrm{s} \theta \mathrm{s} \psi & -\mathrm{c} \psi \mathrm{s} \phi+\mathrm{s} \theta \mathrm{s} \psi \mathrm{c} \phi \\
-\mathrm{s} \theta & \mathrm{c} \theta \mathrm{s} \phi & \mathrm{c} \theta \mathrm{c} \phi
\end{array}\right]
$$

and $\boldsymbol{J}_{2}(\boldsymbol{\eta})$ is the angular velocity transformation matrix defined as

$$
\boldsymbol{J}_{2}(\boldsymbol{\eta})=\left[\begin{array}{ccc}
1 & \mathrm{~s} \phi \mathrm{t} \theta & \mathrm{c} \phi \mathrm{t} \theta \\
0 & \mathrm{c} \phi & -\mathrm{s} \phi \\
0 & \mathrm{~s} \phi / \mathrm{c} \theta & \mathrm{c} \phi / \mathrm{c} \theta
\end{array}\right]
$$

Nonlinear model properties. In Fossen (1994) it is shown that the nonlinear model (1) and (2) satisfies the following model properties

1. $\boldsymbol{M}=\boldsymbol{M}^{\mathrm{T}}>\mathbf{0}$ and $\dot{M}=\mathbf{0}$ (assuming const. added mass for a deeply submerged vehicle).

2. $C(v)=-C^{\mathrm{T}}(v)$ implying that:

$$
x^{\mathrm{T}} C(v) x=0 \forall x \in \mathbb{R}^{6}, \quad v \in \mathbb{R}^{6}
$$

3. $\boldsymbol{v}^{\mathrm{T}} \boldsymbol{D}(\boldsymbol{v}) \boldsymbol{v} \geqslant 0 \forall v \in \mathbb{R}^{6}$.

\section{Appendix B. NPS AUV II model parameters}

The AUV model used in the case studies is based on the Naval Postgraduate School (NPS) AUV II, Healey and Lienard (1993). The horizontal motion (surge, sway and yaw) of the NPS AUV II is described by

$$
\begin{array}{lll}
W=53400 \mathrm{~N} & I_{z}=2038 \mathrm{kgm}^{2} & C_{d}=0.0034 \\
m=5443.4 \mathrm{~kg} & L=5.3 \mathrm{~m} & \rho=1000 \mathrm{~kg} / \mathrm{m}^{3}
\end{array}
$$

The dynamic pressure is

$$
q=0 \cdot 5 \rho L^{2}=14045 \cdot 0 \mathrm{~kg} / \mathrm{m}
$$

whereas

$$
\begin{array}{rlrl}
X_{\dot{u}} & =-7 \cdot 6 \cdot 10^{-3} q L & X_{u|u|} & =-C_{d} q \\
Y_{\dot{v}}=-5 \cdot 5 \cdot 10^{-2} q L & Y_{v} & =-1 \cdot 0 \cdot 10^{-1} q \\
N_{\dot{r}} & =-3 \cdot 4 \cdot 10^{-3} q L^{3} & N_{r} & =-1.6 \cdot 10^{-2} q L^{2} \\
Y_{\dot{r}} & =1 \cdot 2 \cdot 10^{-3} q L & N_{v} & =-7.4 \cdot 10^{-3} q L
\end{array}
$$


The matrices are

$$
\begin{aligned}
\boldsymbol{M} & =\left[\begin{array}{ccc}
m-X_{\dot{u}} & 0 & 0 \\
0 & m-Y_{\dot{v}} & 0 \\
0 & 0 & I_{z}-N_{\dot{r}}
\end{array}\right] \\
\boldsymbol{C}(\boldsymbol{v}) & =\left[\begin{array}{ccc}
0 & 0 & -\left(m-Y_{\dot{v}}\right) v \\
0 & 0 & \left(m-X_{\dot{u}}\right) u \\
\left(m-Y_{\dot{v}}\right) v & -\left(m-X_{u}\right) u & 0
\end{array}\right] \\
\boldsymbol{D}(\boldsymbol{v}) & =\left[\begin{array}{ccc}
-X_{u|u|}|u| & 0 & 0 \\
0 & -Y_{v} & -Y_{r} \\
0 & -N_{v} & -N_{r}
\end{array}\right]
\end{aligned}
$$

Regression form. This model can be written in regression form

$$
M \dot{r}+C(v) r+D(v) r=Y(\dot{r}, r, v) \theta
$$

with parameter vector

$$
\boldsymbol{\theta}=\left[m-X_{u}, m-Y_{\dot{v}}, I_{z}-N_{\dot{r}},-X_{u|u|},-Y_{v},-N_{r},-Y_{r},-N_{v}\right]^{\mathrm{T}}
$$

and regressor

$$
\boldsymbol{Y}=\left[\boldsymbol{Y}_{m}+\boldsymbol{Y}_{c}, \boldsymbol{Y}_{d}\right]
$$

where

$$
\begin{aligned}
\boldsymbol{Y}_{m} & =\left[\begin{array}{ccc}
\dot{r}_{1} & 0 & 0 \\
0 & \dot{r}_{2} & 0 \\
0 & 0 & \dot{r}_{3}
\end{array}\right] \\
\boldsymbol{Y}_{c} & =\left[\begin{array}{ccc}
0 & -r_{2} v_{3} & 0 \\
r_{1} v_{3} & 0 & 0 \\
-r_{1} v_{2} & r_{2} v_{1} & 0
\end{array}\right] \\
\boldsymbol{Y}_{d} & =\left[\begin{array}{ccccc}
\left|r_{1}\right| v_{1} & 0 & 0 & 0 & 0 \\
0 & v_{2} & 0 & v_{3} & 0 \\
0 & 0 & v_{3} & 0 & v_{2}
\end{array}\right]
\end{aligned}
$$

\section{REFERENCES}

Berghuis, H. (1993). Model-Based Robot Control: From Theory to Practice. PhD thesis. University of Twente, Enschede, The Netherlands.

FossEN, T. I. (1993). Comments on Hamiltonian Adaptive Control of Spacecraft. IEEE Transactions on Automatic Control, AC-38, 671-672. 
Fossen, T. I. (1994). Guidance and Control of Ocean Vehicles (John Wiley and Sons Ltd). FOSSEN, T. I. and FJELLSTAD, O. E. (1995). Nonlinear modelling of marine vehicles in 6 degrees of freedom. International Journal of Mathematical Modelling of Systems, JMMS-1.

FosSEn, T. I. and SAGATUN, S. I. (1991). Adaptive control of nonlinear systems: A case study of underwater robotic systems. Journal of Robotic Systems, JRS-8, 393-412.

Gelb, A., Kasper, J. F. JR., Nash, R. A. JR., Price, C. F. and Sutherland, A. A. JR. (1988). Applied Optimal Estimation (MIT Press, Boston, Massachusetts).

HEALEY, A. J. and LIENARD, D. (1993). Multivariable sliding mode control for autonomous diving and steering of unmanned underwater vehicles. IEEE Journal of Ocean Engineering, OE-18, 327-339.

NARENDRA, K. S. and ANNASWAMY, A. M. (1987). A new adaptive law for robust adaption without persistent excitation. IEEE Transactions on Automatic Control, AC-32, 134-145.

SADEGH, N. and HoROwITZ, R. (1990). Stability and robustness analysis of a class of adaptive controllers for robotic manipulators. International Journal of Robotics Research, 9, 74-94.

SAGATUN, S. I. and FosSEN, T. I. (1991). Lagrangian formulation of underwater vehicles' dynamics. In: Proceedings of the IEEE International Conference on Systems, Man and Cybernetics. Charlottesville, VA. pp. 1029-1034.

Slotine, J. J. E. and Di BENEDETTO, M. D. (1990). Hamiltonian adaptive control of spacecraft. IEEE Transactions on Automatic Control, AC-35, 848-852.

SNAME (1950). The Society of Naval Architects and Marine Engineers. Nomenclature for Treating the Motion of a Submerged Body Through a Fluid. In: Technical and Research Bulletin Nos 1-5. 